\title{
A radiation hardened digital fluxgate magnetometer for space applications
}

\author{
D. M. Miles ${ }^{1}$, J. R. Bennest ${ }^{2}$, I. R. Mann ${ }^{1}$, and D. K. Millling ${ }^{1}$ \\ ${ }^{1}$ Department of Physics, University of Alberta, Edmonton, Alberta, T6G 2E1, Canada \\ ${ }^{2}$ Bennest Enterprises Ltd, Summerland, British Columbia, V0H 1Z0, Canada \\ Correspondence to: D. M. Miles (dmmiles@ualberta.ca) \\ Received: 5 February 2013 - Published in Geosci. Instrum. Method. Data Syst. Discuss.: 20 February 2013 \\ Revised: 30 July 2013 - Accepted: 28 August 2013 - Published: 13 September 2013
}

\begin{abstract}
Space-based measurements of Earth's magnetic field are required to understand the plasma processes responsible for energising particles in the Van Allen radiation belts and influencing space weather. This paper describes a prototype fluxgate magnetometer instrument developed for the proposed Canadian Space Agency's (CSA) Outer Radiation Belt Injection, Transport, Acceleration and Loss Satellite (ORBITALS) mission and which has applications in other space and suborbital applications. The magnetometer is designed to survive and operate in the harsh environment of Earth's radiation belts and measure low-frequency magnetic waves, the magnetic signatures of current systems, and the static background magnetic field. The new instrument offers improved science data compared to its predecessors through two key design changes: direct digitisation of the sensor and digital feedback from two cascaded pulse-width modulators combined with analog temperature compensation. These provide an increase in measurement bandwidth up to $450 \mathrm{~Hz}$ with the potential to extend to at least $1500 \mathrm{~Hz}$. The instrument can resolve $8 \mathrm{pT}$ on a $65000 \mathrm{nT}$ field with a magnetic noise of less than $10 \mathrm{pT} / \sqrt{\mathrm{Hz}}$ at $1 \mathrm{~Hz}$. This performance is comparable with other recent digital fluxgates for space applications, most of which use some form of sigma-delta $(\Sigma \Delta)$ modulation for feedback and omit analog temperature compensation. The prototype instrument was successfully tested and calibrated at the Natural Resources Canada Geomagnetics Laboratory.
\end{abstract}

\section{Introduction and application}

Space-based measurements of Earth's magnetic field are required to understand the plasma processes underlying the solar-terrestrial connection. This includes the nature of the current systems and plasma waves during storms and substorms which are thought to cause acceleration and loss of energetic particles in the Van Allen radiation belts, and which constitute space weather. This paper describes a radiation hardened fluxgate magnetometer developed for the proposed Canadian Space Agency's (CSA) Outer Radiation Belt Injection, Transport, Acceleration and Loss Satellite (ORBITALS) mission (Mann et al., 2006).

The base fluxgate design used in this prototype has more than two decades of terrestrial heritage in the Canadian and American CARISMA/CANOPUS (Mann et al., 2008), POLARIS (Eaton et al., 2005), and EMScope/EarthScope USArray (Schultz, 2009) instruments built by Narod Geophysics Ltd (NGL) (e.g. Narod and Bennest, 1990). The NGL design was previously modified for low-radiation space applications as the magnetic field instrument (MGF) in the CSA's enhanced Polar Outflow Probe (ePOP) payload on the CAScade, Smallsat and IOnospheric Polar Explorer (CASSIOPE) satellite (Wallis et al., 2006).

Magnetic measurements for ORBITALS are technically challenging as the instrument must be capable of resolving small field variations in the presence of the large spin-tone signal caused by the satellite's spin ( $10 \mathrm{~s}$ period) in the frame of Earth's magnetic field. These measurements must be made while surviving and operating within Earth's radiation belts. In 2011, in addition to consideration for ORBITALS, the instrument development was continued for an additional 
Table 1. Performance characteristics of relevant recent and historical spaceflight fluxgate magnetometers.

\begin{tabular}{|c|c|c|c|c|c|c|}
\hline Mission & $\begin{array}{l}\text { Max B } \\
( \pm \mathrm{nT})\end{array}$ & $\begin{array}{l}\text { Resolution } \\
\text { (nT) }\end{array}$ & $\begin{array}{l}\text { Rate } \\
\text { (sps) }\end{array}$ & $\begin{array}{l}\text { Noise } \\
(\mathrm{pT} / \sqrt{\mathrm{Hz}}) \\
\text { at } 1 \mathrm{~Hz}\end{array}$ & $\begin{array}{l}\text { Power } \\
\text { (W) }\end{array}$ & Reference \\
\hline MJS'77 - High & 50000 & 8 ranges & 25 & 1000 & $0.12 /$ axis & Acuña (1974) \\
\hline MJS'77 - Low & 2000000 & 2 ranges & 25 & 13 & 0.09/axis & Acuña (1974) \\
\hline Pioneer XI & 1730000 & 338 & 0.08 & unknown & 0.3 & Acuña and Ness (1973) \\
\hline CRRES & $45000 / 850$ & $22 / 0.4$ & 16 & unknown & unknown & Singer et al. (1992) \\
\hline Astrid-2 & 61865 & 0.118 & 2048 & unknown & unknown & Pedersen et al. (1999) \\
\hline AMPTE/IRM & $60000 / 4000$ & $1.8 / 0.12$ & 32 & 25 & 6.9 & Luehr et al. (1985) \\
\hline CASSIOPE/ePOP & 65536 & 0.0625 & 160 & 7 & 1.1 & Wallis et al. (2006) \\
\hline Proto - DAWN & 2000 & 70 & 144 & 7 & unknown & Magnes et al. (2003) \\
\hline DoubleStar & $32764 / 128$ & $4 / 0.016$ & 22 & 5 & 3.6 & Carr et al. (2005) \\
\hline Proto - Imperial & 327 & 0.01 & $22 / 122$ & 10 & unknown & O'Brien et al. (2007) \\
\hline Proto - SMILE & 50000 & 0.1 & 250 & 30 & 0.33 & Forslund et al. (2008) \\
\hline Proto - ASIC & 60000 & 0.014 & 128 & 8 & 0.06 & Magnes et al. (2008) \\
\hline THEMIS & 25000 & 0.003 & 128 & 10 & 0.8 & Auster et al. (2008) \\
\hline
\end{tabular}

application as a secondary science payload called as the Plasma and Radiation In Molniya Orbit (PRIMO) secondary science payload (Mann et al., 2011) on the CSA's Polar Communications and Weather (PCW) satellite (Trishchenko and Garand, 2012).

For both missions, the fluxgate magnetometer was required to measure the static magnetic field and magnetic waves at frequencies below $10 \mathrm{~Hz}$. The static magnetic field measurements are required to understand the structure of the magnetospheric magnetic field, the role of current systems, and the dynamical motion and wave-particle acceleration, transport, and loss of energetic particles. Ultra low frequency (ULF) magnetic waves are thought to interact with low-energy particle populations and accelerate them to form damaging, high energy $(\mathrm{MeV})$ space radiation (e.g. Friedel et al., 2002). Wave-particle interactions are also believed to be responsible for scattering particles into the loss cone. This depletes the radiation belts and has potentially important consequences for the atmosphere and climate change (e.g. Seppälä et al., 2007).

Current fluxgate sensor technology provides limited useful sensitivity at high ( $>10$ to $100 \mathrm{~Hz}$ ) frequencies compared to induction coil magnetometers. However, if future cores are available with substantially lower noise (e.g. Narod, 2013) then higher frequency fluxgate measurements would become increasingly useful. Also, in applications such as flights of opportunity on commercial satellites, it may not be possible to fly both fluxgate and induction coil magnetometers. In this case, even lower sensitivity high frequency measurements may be useful.

The prototype instrument uses only electronic components which have space grade (Class $\mathrm{S}$ ) equivalents that are radiation tolerant to at least $100 \mathrm{krad}$. This allows the instrument to be manufactured for space flight applications in a high radiation environment. The new instrument has a low parts count and is not dependent on high-performance commercial components. This has been achieved by replacing much of the analog signal conditioning used in previous designs with equivalent digital processing in a field programmable gate array (FPGA).

The new design is simpler than its predecessors and offers improved science data through two key design improvements. Firstly, direct digitisation of the sensor removes the need for complex analog filtering of the fluxgate signal. Secondly, a novel dual pulse width modulation design provides digital feedback while preserving the sensor temperature compensation found in classical analog fluxgate magnetometers. These two changes provide bandwidth up to $450 \mathrm{~Hz}$ with the potential to extend to at least $1500 \mathrm{~Hz}$ in future work. This paper describes the instrument improvements, the key subsystems, and the performance of the resulting prototype instrument.

\section{Modern state of space fluxgate magnetometers}

The new fluxgate magnetometer design for the ORBITALS application was designed to meet the mission's science requirements and survive the expected harsh radiation environment of the Van Allen radiation belts. The prototype described in this paper meets these requirements and is an interim step towards a fully modernised radiation hardened digital instrument. Table 1 summarises the key performance metrics of recent and historical fluxgate magnetometers designed for space applications.

The MGF instrument from the CSA's ePOP payload (Wallis et al., 2006) is the design on which this prototype is based but is not sufficiently radiation tolerant for the ORBITALS mission.

O'Brien et al. (2007) provides a recent example of replacing analog circuitry with digital processing. However, the 
resulting prototype has limited range and does not actively temperature compensate the sensor.

NASA's THEMIS fluxgate magnetometer (Auster et al., 2008) is a recently deployed, and highly successful, spaceflight instrument. However, the THEMIS instrument uses component level embedded shielding which is not considered acceptable in the harsh radiation environment of the ORBITALS mission. This approach to radiation mitigation tends to have geometric vulnerabilities (e.g. vulnerable to omnidirectional radiation). The ORBITALS mission has long dwells in the highly energetic radiation belts and forbid the use of this type of radiation mitigation. ORBITALS planned to use $7 \mathrm{~mm}$ of $\mathrm{Al}$ shielding at the instrument level, so radiation reaching the components is necessarily highly penetrating and is not effectively stopped by the integrated shielding.

SMILES (Forslund et al., 2008) is a miniaturised digital fluxgate magnetometer designed for small spacecraft applications. This design uses a sophisticated hybrid of pulse width modulation (PWM) and sigma-delta $(\Sigma \Delta)$ modulation to provide digital feedback. The hybrid design seeks to balance the excellent linearity of a PWM with the higher usable bandwidth of $\Sigma \Delta$ modulation. The feedback itself is not temperature compensated, rather the temperature of the sensor is measured for post-processed compensation. The prototype has outstanding mass and volume characteristics; however, it relies on commercial off-the-shelf components which are not suitable for use in the radiation belts.

Magnes et al. (2008) describe a highly integrated front-end for a spaceborne fluxgate. The design is very compact, low power and has an excellent parametric radiation tolerance of $170 \mathrm{krad}$ (300 krad functional). The front-end is implemented in a custom hybrid analog/digital application specific integrated circuit (ASIC). The design merges a second order $\Sigma \Delta$ modulator with a conventional analog fluxgate control loop. The design uses a resistor for feedback current translation and achieves excellent drift and temperature characteristics.

The prototype described in this paper implements a new digital feedback topology using two cascaded PWMs combined with classical analog temperature compensation of the feedback circuit. This design has lower complexity than many of the state-of-the art instruments and can be constructed from highly radiation tolerant parts. This simplicity is at the expense of being larger, less integrated, and consuming more power. The cascaded PWMs have the potential for non-linearity depending on the precision of the mixing network.

\section{Instrument design}

The instrument described in this paper is a redesign and further radiation hardened development of the CASSIOPE/ePOP fluxgate magnetometer instrument (MGF); analog signal conditioning circuitry was replaced with digital processing in an FPGA to mitigate radiation and temperature

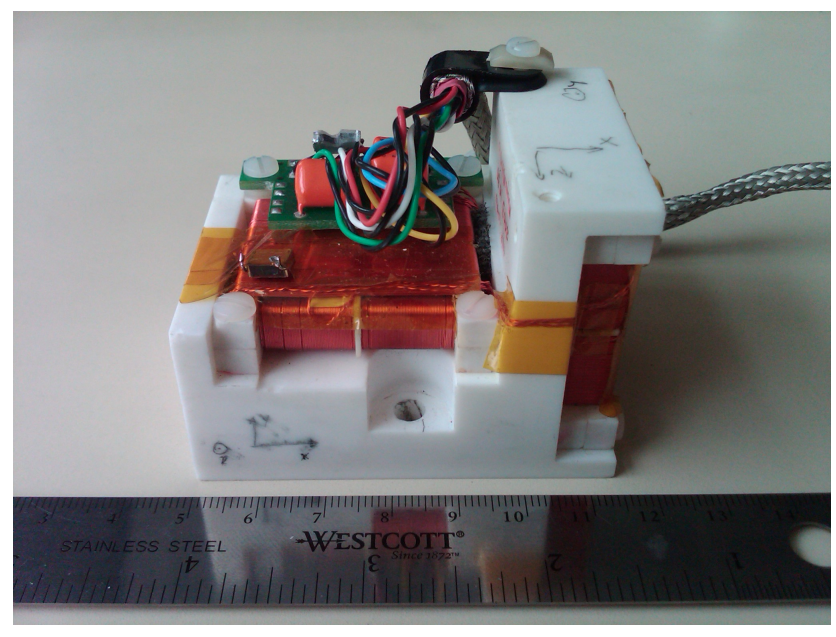

Fig. 1. Fluxgate sensor used in the prototype instrument. This hardware is the CASSIOPE/ePOP MGF engineering model with minor modifications.

effects. The prototype uses a modified version of the flightspare fluxgate sensor from the CASSIOPE/ePOP with two low-noise Infinetics ring cores (Fig. 1). This sensor uses the established technique (e.g. Acuña and Pellerin, 1969) of double winding a ring core to sense two components of the magnetic field using one ring core.

The ePOP MGF electronics were designed such that the noise floor of the overall instrument was set by the intrinsic noise of the two ring cores in the sensor. One of the goals for the new prototype instrument was to achieve the same noise floor of less than $10 \mathrm{pT} / \sqrt{\mathrm{Hz}}$ root mean square (RMS) at $1 \mathrm{~Hz}$ as was achieved by the CASSIOPE/ePOP fluxgate but in a radiation hard, digital, and temperature compensated design. As in the MGF instrument sensor, the $\boldsymbol{X}$ and $\boldsymbol{Y}$ components are derived from a single sense winding on each core while the $\boldsymbol{Z}$ component is derived from sense windings on each sensor core connected in series (see Fig. 2). The two dualwound sensor bobbins are mounted on a block of MACOR machinable ceramic to minimise sensitivity changes due to temperature variation.

Figure 3 shows a single component block diagram of the new instrument. The prototype uses magnetic feedback to null the majority of the magnetic field inside the sensor. The measurement of the ambient field is then the sum of the applied magnetic feedback and the measured small residual field in the sensor. An FPGA controller generates a $28800 \mathrm{~Hz}$ drive signal which is power amplified (PA) and sent into the drive winding to periodically saturate and unsaturate the ring cores. The direction of saturation is alternated to avoid magnetising the core. The modulated core permeability creates a fluxgate signal for each magnetometer component corresponding to the magnetic field strength inside the sensor in that axis. The current output from the sensor is converted to a voltage $(\mathrm{I} / \mathrm{V})$ and is sampled by the analog-to-digital 


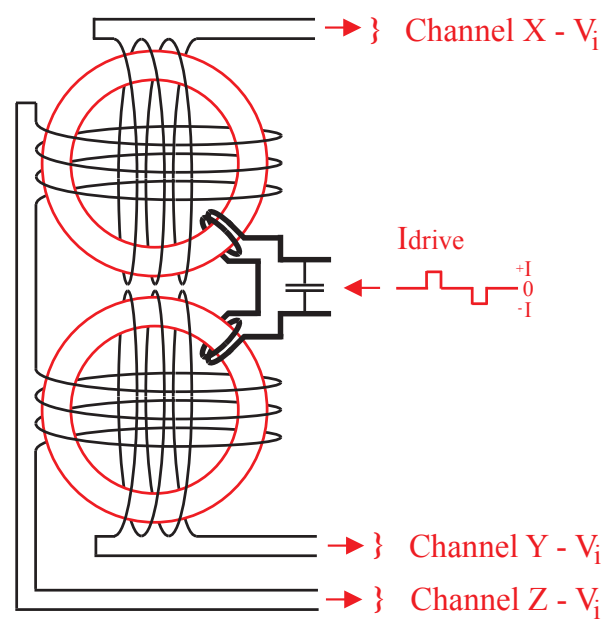

Fig. 2. Schematic wiring of the $\boldsymbol{X}, \boldsymbol{Y}$, and $\boldsymbol{Z}$ sensor components and the drive winding in the prototype. $\boldsymbol{X}$ and $\boldsymbol{Y}$ are single windings while $\boldsymbol{Z}$ is derived from two windings in series. Note that the two ring cores are physically orthogonal as shown in Fig. 1.

converter (ADC) to become the input to a control loop implemented in the FPGA. The output of the control loop is sent to the digital-to-analog converter (DAC) and converted into a precise, temperature compensated current (V/I). This negative feedback drives each component of the magnetic field in the sensor head towards zero.

Figure 4 shows a block diagram of the major elements of the FPGA logic. Variables $k_{0}, k_{1}, k_{2}$, and $k_{3}$ are experimentally determined scaling coefficients for the coarse PWM, fine PWM, ADC, and offset respectively. The ADC value used to compute the final datum is the arithmetic mean of 64 ADC samples. Averaging over an even number of ADC reading and subtracting an experimentally determined constant removes the static offset which would otherwise result from the coupling of the drive winding to the sense winding.

\subsection{Direct digitisation}

The bandpass filters and analog integrators traditionally used in second harmonic fluxgate magnetometers (e.g. Narod and Bennest, 1990) to sense the fluxgate signal at twice the sensor drive frequency $(2 f)$ are not required in this design. In the prototype, the sensor is directly digitised by sampling the instantaneous output from the sensor. In a classical design, the sensor is bandpass filtered, and integrated using analog processing before the signal is digitised. The direct digitisation approach can be described using the standard fluxgate induction equation (Ripka, 2001) which relates the sensor voltage $V_{i}$ to the static magnetic $\boldsymbol{H}$ field through the modulation of the relative permeability $\mu_{\mathrm{r}}$ of the sensor core:

$V_{i}=\left(N A \mu_{0} \boldsymbol{H}\right) \frac{\mathrm{d} \mu_{\mathrm{r}}}{\mathrm{d} t}$.

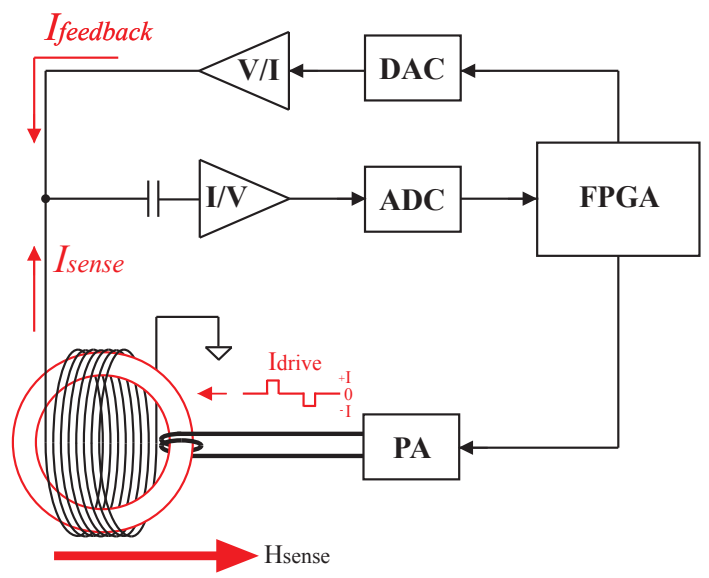

Fig. 3. Schematic of one fluxgate component showing the major components. The FPGA controller generates a $28800 \mathrm{~Hz}$ drive signal that is power amplified (PA) and sent into the drive winding to periodically saturate and and unsaturate the ring cores. The bandpass, phase synchronous detector, low pass, and integrator hardware in a classic $2 f$ fluxgate are replaced with a current-to-voltage converter (I/V) and an ADC to digitise the signal. An FPGA uses the fluxgate signal as the input to a control loop to drive the magnetic field in the sensor towards zero. The output of the control loop is converted to an analog signal (DAC) and then to a highprecision, temperature compensated current to provide magnetic feedback (V/I).

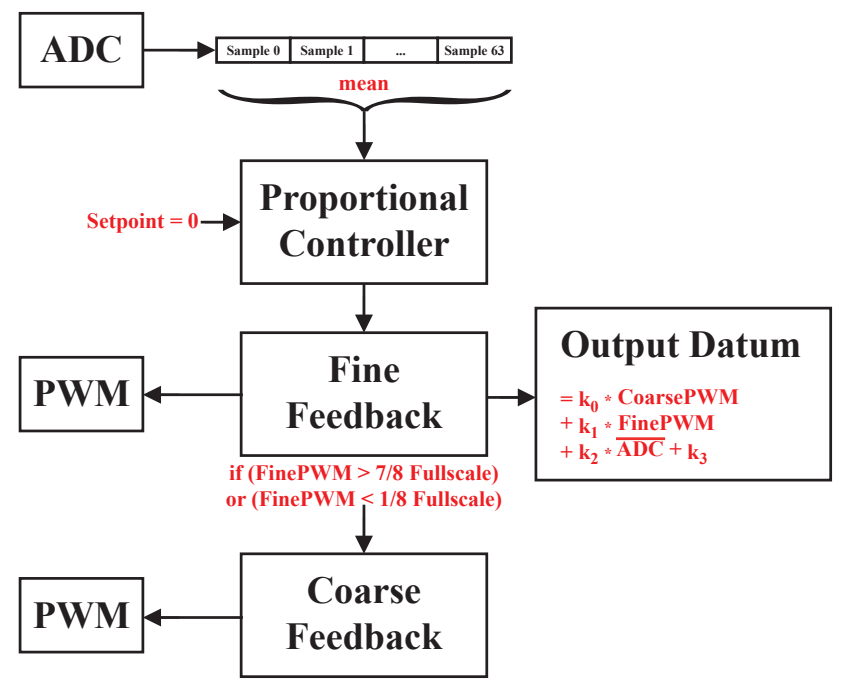

Fig. 4. Block diagram showing major elements of the FPGA logic. Variables $k_{0}, k_{1}, k_{2}$, and $k_{3}$ are experimentally determined scaling coefficients for the coarse PWM, fine PWM, ADC, and offset respectively.

The sense coil is held in the short-circuit configuration shown in Fig. 5 using a virtual ground from the inverting input of an operational amplifier. The sense winding has a spike in sensitivity corresponding to the natural self resonance of the coil. Holding the coil in a short circuit helps linearise the 


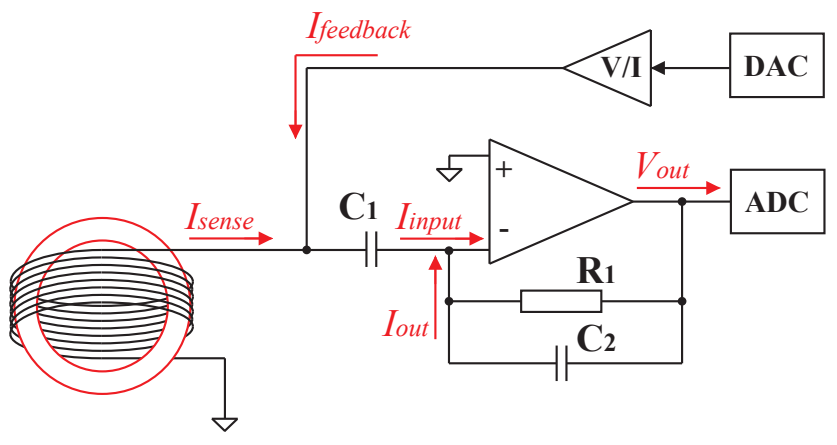

Fig. 5. Equivalent circuit of the sensor preamplifier in the prototype instrument.

coil response by suppressing this self resonance. The voltage across the coil is forced to zero by the short circuit; however, there is still a circulating current determined by Ohm's law where $V_{i}$ is the unloaded coil voltage.

$I_{\text {sense }}=\frac{V_{\mathrm{i}}}{R_{\text {winding }}}$

Substituting the fluxgate induction equation from Eq. (1) gives

$I_{\text {sense }}=\frac{\left(N A \mu_{0} \boldsymbol{H}\right) \frac{\mathrm{d} \mu_{\mathrm{r}}}{\mathrm{d} t}}{R_{\text {winding }}}$.

The operational amplifier is configured as a transimpedance amplifier (current-to-voltage converter) and is selected to be approximated by the ideal amplifier assumptions (zero input current, zero offset, arbitrarily large gain). As the net current at the inverting input must be zero, Kirchhoff's current law analysis at the inverting amplifier input shows that

$I_{\text {net }}=I_{\text {sense }}+I_{\text {input }}+I_{\text {out }}=0$.

The input current to the amplifier is essentially zero $\left(I_{\text {input }}=0\right)$. The capacitor $C_{1}$ is selected such that it passes the second harmonic AC fluxgate signal but blocks the quasistatic signal from the feedback DAC so $I_{\text {feedback }}=0$ at the $b$ frequency (twice the drive frequency, $f$ ). $C_{2}$ keeps the amplifier from oscillating and suppresses high frequency noise but doesn't pass significant current at the $2 f$ frequency. $I_{\text {out }}$ can be re-written using Ohm's law $\left(I_{\text {out }}=V_{\text {out }} / R_{1}\right)$. Making these substitutions gives

$0=I_{\text {sense }}+I_{\text {input }}+I_{\text {out }}+I_{\text {feedback }}$,

$0=I_{\text {sense }}+0+\frac{V_{\text {out }}}{R_{1}}+0$,

$V_{\text {out }}=-R_{1} \cdot I_{\text {sense }}$.

$I_{\text {sense }}$ from the fluxgate sensor is balanced by $I_{\text {out }}$ from the amplifier feedback. Finally, substitution for $I_{\text {sense }}$ using Eq. (3) gives

$V_{\text {out }}=-\frac{R_{1}}{R_{\text {winding }}} N A \mu_{0} \boldsymbol{H} \frac{\mathrm{d} \mu_{\mathrm{r}}}{\mathrm{d} t}$.

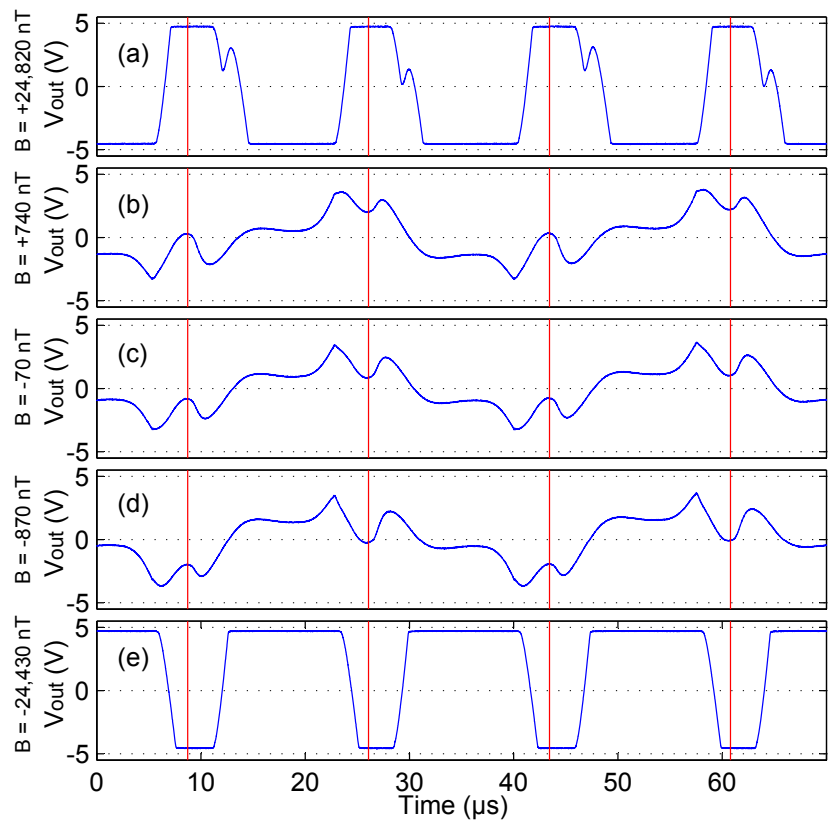

Fig. 6. $V_{\text {out }}$ at various applied magnetic fields that are (a) large positive $(+24820 \pm 10 \mathrm{nT})$, (b) small positive $(+740 \pm 10 \mathrm{nT})$, (c) near zero $(-70 \pm 10 \mathrm{nT})$, (d) small negative $(-870 \pm 10 \mathrm{nT})$, and (e) large negative $(-24430 \pm 10 \mathrm{nT})$.

When the drive winding is modulating the relative permeability of the sensor $\left(\frac{d \mu_{\mathrm{r}}}{\mathrm{d} t} \neq 0\right)$ the preamplifier produces a voltage $V_{\text {out }}$. This voltage is proportional to the instantaneous magnetic $\boldsymbol{H}$ field, has configurable gain via resistor $R_{1}$, and provides a low-impedance output which can be digitised without loading the sensor and distorting the signal.

The $V_{\text {out }}$ signal is composed of even harmonics of the $\frac{\mathrm{d} \mu_{\mathrm{r}}}{\mathrm{d} t}$ fluxgate action frequency at $2 f, 4 f$, etc. and the transformer coupled harmonics of the drive signal at $f, 2 f$, etc. The fluxgate action will cause $V_{\text {out }}$ to pulse at $2 f$ in proportion to the strength of the local magnetic field. However, these pulses will be superimposed on the constant, phase locked residual harmonics of the drive signal.

Figure 6 shows a time series of $V_{\text {out }}$ under test conditions using applied external magnetic fields which are (a) large positive $(+24820 \pm 10 \mathrm{nT}), \quad$ (b) small positive $(+740 \pm 10 \mathrm{nT})$, (c) near zero $(-70 \pm 10 \mathrm{nT})$, (d) small negative $(-870 \pm 10 \mathrm{nT})$, and (e) large negative $(-24430 \pm 10 \mathrm{nT})$. Panels a and e are saturated due to maximum amplifier output compliance and do not correspond the external magnetic field beyond indicting out-of-range high and low respectively. However, in the operating instrument the digital feedback would quickly return the field within the sensor back into the unsaturated region.

The data was collected by disabling magnetic feedback, placing the sensor in a solenoid within a magnetic shield, and applying various magnetic field strengths $(\boldsymbol{B})$ using the solenoid and a precision current source. The vertical red lines 
in Fig. 6 show the times when the ADC samples $V_{\text {out }}$, phase locked to the $2 f$ signal effectively creating a synchronous detector. The irregularity on the decreasing slope in Fig. 6a $(+24820 \pm 10 \mathrm{nT})$ varies by axis and individual sensor. It is believed to be related to asymmetries in the sensor core.

The amplitude of the $2 f$ pulses, as measured at the ADC trigger points, tracks the applied magnetic field in amplitude and polarity. However, even in a near zero field (Fig. 6c, $-70 \pm 10 \mathrm{nT}$ ), there is a residual AC signal from the drive current primarily at $f$ and $2 f$. The ADC measurements must be averaged over an even number of samples to cancel the harmonics.

Digitising the signal directly at the ADC trigger points requires minimal analog processing and is not subject to temperature effects common to analog filters. However, since the signal has not been bandpass filtered around the $2 f$ frequency, low frequency fluctuation of the amplifier and the $\frac{\mathrm{d} \boldsymbol{H}}{\mathrm{d} t}$ action of the sensor will be added to the fluxgate signal. This represents a potential source for both noise and zero offset temperature dependence.

It is important to note that even in a strong field (Fig. 6a, $+24820 \pm 10 \mathrm{nT}$ and Fig. $6 \mathrm{e},-24430 \pm 10 \mathrm{nT}$ ) the amplitude of the error signal at the ADC trigger points is monotonic and strictly increasing with magnetic field. This is essential to the control system because any local extrema or out-of-range polarity inversion would cause the control loop to apply feedback in the wrong direction and cause the instrument to oscillate or become stuck and full at positive or negative full scale. Signal inversion can be possible with certain amplifiers with large signals such that the inputs or outputs approach the supply voltages. This would not be a problem during normal operation with the digital feedback enabled but could interfere with the initial servo to the instrument's operating point when initially turned on in a field large enough to clip the analog system.

\subsection{Analog-to-digital converter oversampling}

The digital feedback is always trying to drive the field in the sensor to zero so the output of the sensor is both a partial measurement of the field and the instantaneous error in the digital feedback value. The sensor output is therefore referred to as the "error signal" to be consistent with control system nomenclature.

The prototype instrument is designed such that the analogto-digital converter has a least significant bit of $57 \mathrm{pT}$. This is the smallest resolution which can accommodate the analog error signal under normal operating conditions. However, the error signal is digitised at $57600 \mathrm{sps}$ and decimated by 64 into a $900 \mathrm{sps}$ (samples per second) data product. Oversampling by four times can create one effective bit of resolution. Therefore, oversampling by $N=64=4^{3}$ creates three additional bits of resolution for an effective resolution of

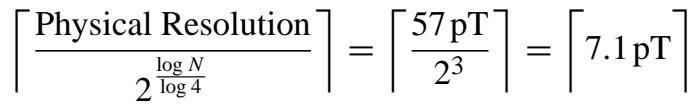

or, to the next largest integer, $8 \mathrm{pT}$ resolution.

In the current prototype the $57600 \mathrm{sps}$ measurements are simply decimated into the final data product. This makes the prototype vulnerable to aliasing effects from out-of-band signals. In future work a digital antialiasing filter will be added to mitigate this.

\subsection{Temperature compensated digital feedback}

In this instrument, the PWM based DAC changes its average output by varying the duty cycle of a pulse train emitted at a fixed repetition rate. A subsequent low-pass filter (LPF) attenuates the repetition-rate frequency, and its harmonics, leaving only the average value. The simplest possible PWM based DAC feedback system has three components.

1. A single-pole, double-throw (SPDT) break-beforemake transmission-gate switch (sourced from precision bipolar reference voltages) to create a variable duty cycle pulse train.

2. A low-pass filter to remove the switching frequency.

3. A voltage-to-current converter to drive the feedback coil.

Some digital fluxgate magnetometers (e.g. O'Brien et al., 2007) use a simple resistor-capacitor low-pass filter to drive the digital pulse train into the feedback coil using a resistor for current conversion. To first order, the resistor acts as a voltage-to-current converter and the average feedback current experienced by the sensor is the same.

However, a simple resistor is not a controlled current source and cannot be used to provide temperature compensation for the sensor. The geometry of the sensor's feedback winding will change slightly with temperature primarily due to variation in coil length and circumference. As a consequence, the amount of magnetic feedback for a given current varies with temperature. Primdahl (1970) and then Acuña et al. (1978) describe a technique for temperature compensation by noting that the resistance of the coil also changes with temperature and that these two effects can be used to cancel each other.

In this prototype, a transconductance amplifier (i.e. voltage-to-current converter) is used to provide feedback current. However, the transconductance amplifier is intentionally unbalanced so that the voltage-to-current conversion factor depends on the coil resistance. This dependence on coil resistance is then tuned until the temperature effects of the coil resistance and the coil geometry are equal and opposite.

This temperature compensation technique has been widely used in analog fluxgate magnetometers (e.g. Narod and Bennest, 1990); however, it has been missing in previous 
digital designs. The technique requires a low-pass reconstruction filter on the digital feedback to avoid clipping the transconductance feedback amplifier with the comparatively large switching signal from the PWM. The design outlined below can be constructed from radiation hardened components and implements high resolution digital feedback while preserving the temperature compensation of the fluxgate sensor.

The feedback frequency range should generally be wide enough to include the sampling frequency to make the feedback control loop robust and stable. The prototype instrument measures each axis at $900 \mathrm{sps}$ (samples per second). The PWM repetition rate was set a decade higher to allow a 3 -pole low-pass reconstruction filter with $-3 \mathrm{~dB}$ corner near $1 \mathrm{kHz}$ to provide greater than $60 \mathrm{~dB}$ attenuation of the PWM repetition frequency. This leaves a residual PWM amplitude small enough to avoid saturating the feedback amplifier. The repetition rate of the PWM was set to $14400 \mathrm{~Hz}$ (the next divisor of the common clock frequency) to ensure that the feedback is locked in phase with the other instrument functions.

The magnetic range of the instrument was set to $\pm 65536 \mathrm{nT}$ to cover the expected geomagnetic field experienced in ground and space applications. A resolution of $8 \mathrm{pT}$ was selected in anticipation of ultra-low-noise cores which may result from the ongoing research (e.g. Narod, 2013).

Resolving $8 \mathrm{pT}$ within this range requires 24 bit resolution. The best available radiation hard ADC (the RAD1419) can provide 14 bits of resolution. Even in a constant field, the value of two successive ADC values can vary by more than half the $\pm 2.5 \mathrm{~V}$ total ADC input range due to the significant residual of the sensor drive signal. If the input value is too large or too small then the half of the samples will be clipped and invalid. This restricts the accurately usable input range of the ADC and costs two bits for an effective 12 bits of resolution. This requires the remaining 12 bits of resolution to be supplied by the digital-to-analog converter in the feedback network. Note that although only 12 bits of resolution are required, the digital-to-analog converter must be quiet at the 24 bit level in the frequency range of the instrument to avoid being the dominant noise source in the error signal. In a PWM based digital-to-analog converter, this primarily involves suppressing the PWM repetition rate below the 24 bit level in the final data product.

The base frequency required for a simple PWM to deliver this would be

$14400 \mathrm{~Hz} \times 2^{12}=58982400 \mathrm{~Hz} \approx 60 \mathrm{MHz}$.

This is significantly faster than the best available radiation hardened analog switches (Intersil part HS303ARH) which, in this prototype, started to have marginal performance at $20 \mathrm{MHz}$.

Figure 7 shows a functional diagram of a dual PWM feedback network which solves this problem. Each 10 bit PWM sets its output level by the duty cycle of the pulse train. The low-pass filter reduces the amplitude of the $14400 \mathrm{~Hz}$ PWM

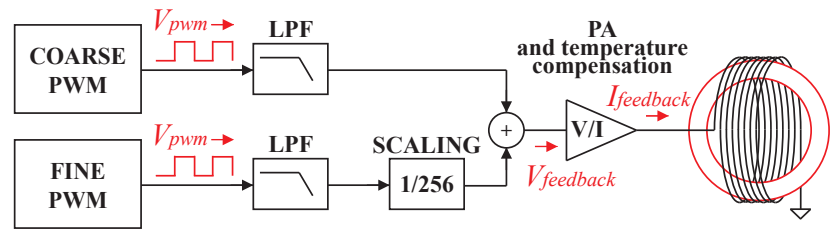

Fig. 7. Block diagram of the dual pulse width modulation feedback network of the prototype instrument. The output is built up from two 10 bit PWMs at $14400 \mathrm{~Hz}$ and low-pass filtered at $1 \mathrm{kHz}$. The two PWMs are scaled to overlap by two bits, summed, and converted into a feedback current using an intentionally mis-balanced transconductance amplifier to temperature compensate the feedback coil.

signal. The voltage of the fine PWM is attenuated to set its scaling compared to the coarse PWM and provide two bits of overlap for the control system. The fine PWM is then summed with the coarse PWM to create a voltage that can be set with a $10+10-2=18$ bit resolution albeit with imperfect linearity as determined by the accuracy of the mixing network.

Ultra-high stability, ultra-low temperature coefficient bulk foil resistors are used in the attenuator, summing network, and the transconductance amplifier feedback networks. Combining two digital-to-analog converters can introduce nonlinear output. This effect is minimised by using resistors which are vendor trimmed to $0.01 \%$ accuracy. Both PWMs are continuously mixed to create the feedback value. This leads to a short transient ripple if, for example, the coarse and fine PWMs each make an equal but opposite adjustment. Although the nominal total feedback remains constant, the two changes in duty cycle take slightly different times to settle through the mixing and filter network resulting in a short transient in the feedback experienced by the fluxgate sensor.

The low-pass reconstruction filters do not completely suppress the $14400 \mathrm{~Hz}$ repetition rate. However, the amplitude is small enough that a classical analog transconductance power amplifier can be used to convert the voltage output into a temperature compensated current source. This approach allows a radiation hardened implementation of both digital feedback and sensor temperature compensation.

\section{Potential for high frequency measurements}

The short-circuit coil and transimpedance amplifier topology used in the prototype is functionally equivalent to the preamplifier topology used by Primdahl et al. (1994) in a paper on high frequency fluxgate performance. In that paper, Primdahl used a more conventional digital switch synchronous detector and analog integrator; however, the functionality is very similar to that in this prototype digital instrument. Primdahl reported that the $-3 \mathrm{~dB}$ point in the amplitude response of the instrument occurred at approximately $1500 \mathrm{~Hz}$, although this 
was tentatively attributed to the synchronous detector rather than the sensor or the fluxgate action.

Primdahl's results, and the fact that there is no obvious physical reason why the fluxgate action should decay with frequency, suggests that the AC response of the prototype instrument should be explored. The repetition rate of the feedback PWMs limits the prototype instrument to $900 \mathrm{sps}$ or $450 \mathrm{~Hz}$ Nyquist. Testing the performance of the instrument above this frequency therefore required probing the analog preamplifier signal directly.

The feedback network of the instrument was temporarily disabled by fixing both the coarse and fine PWM at mid range. In this configuration, the error signal from the preamplifier is analogous to a standard amplitude modulated sinusoidal signal (cf. AM radio). The $2 f$ signal replaces the carrier (frequency $\omega_{\mathrm{c}}$, amplitude $A_{\mathrm{c}}$ ) and the applied magnetic field acts as the modulating signal (frequency $\omega_{\mathrm{m}}$, amplitude $\left.A_{\mathrm{m}}\right)$. The result is a time series of the form:

$y(t)=A_{\mathrm{c}} \times \sin \left(\omega_{\mathrm{c}} t\right)+\frac{A_{\mathrm{m}}}{2}\left[\sin \left(\omega_{\mathrm{c}}+\omega_{\mathrm{m}}\right) t+\sin \left(\omega_{\mathrm{c}}-\omega_{\mathrm{m}}\right) t\right]$.

This analogy allows us to visualise the fluxgate action directly by sampling the error signal with a bench-top spectrum analyser. The $2 f$ carrier frequency shows as a spectral feature at $57600 \mathrm{~Hz}$ whose amplitude is dependent on the static field strength. However, an applied AC test signal will appear as two sideband carriers at $57600 \mathrm{~Hz}$ plus or minus the $\mathrm{AC}$ signal frequency. The panels in Fig. 8 show, from top to bottom, the resulting spectral plots for no test signal, 100, 200, 500,1000 , and $1500 \mathrm{~Hz}$. Note that the amplitude of the sideband carriers is essentially constant with frequency increasing slightly at $1500 \mathrm{~Hz}$ and there are no other large amplitude features within $57600 \pm 1500 \mathrm{~Hz}$. This suggests that, if the PWM frequency and sampling frequency were increased, the prototype fluxgate should provide data up to at least $1500 \mathrm{~Hz}$.

\section{Instrument performance}

\subsection{Test set-up}

Magnetic noise and RMS error analysis were conducted at the National Resources Canada Geomagnetics (NRCan) Laboratory on Anderson Rd, Ottawa, ON, Canada. The Building 8 facility contains a calibrated reference source, a three-axis Helmholtz coil, and an observatory grade magnetometer to cancel local geomagnetic variations. This provides a magnetically quiet location suitable for the long-period measurements required to characterise the low-frequency noise floor of the instrument.

Instrument resolution and $\mathrm{AC}$ performance were completed at the University of Alberta CARISMA laboratory in Edmonton, AB, Canada. The fluxgate sensor was placed in a test solenoid within a three layer magnetic shield. The test signals were generated by driving the solenoid with a Stanford Research DS360 ultra low distortion function generator. This facility has significantly more magnetic noise than the NRCan facility (nearby elevator, large compressor for a walk-in freezer, and overhead air-conditioning and ventilation fans) which make noise measurements, particularly at low frequencies, difficult compared to the NRCan facilities. The test solenoid and signal generator are not calibrated against an established magnetic reference. However, the CARISMA laboratory test fixture was developed for testing induction coil magnetometers and is ideal for testing the dynamic performance of the prototype fluxgate.

\subsection{Magnetic resolution}

The magnetic resolution of the coupled fluxgate sensor and electronics were validated using test signals applied by a solenoid within a three layer magnetic shield. Figure 9 shows a time series of two cycles, each of square waves at amplitudes of $1,0.5,0.25$, and $0.125 \mathrm{nT}$ respectively. Data is postprocessed with a $1 \mathrm{~Hz}$ low-pass filter to remove environmental $60 \mathrm{~Hz}$ noise.

Figures 10 and 11 show amplitude spectra resolving $18 \mathrm{pT}$ RMS (50 pT peak-to-peak) sine-wave test signals at 1 and $400 \mathrm{~Hz}$, respectively. An 18 pT RMS test signal, rather than 8 pT RMS, was used such that the contribution of the noise floor to the spectral amplitude measurement was negligible. The narrow spectral features near 11,55 , and $60 \mathrm{~Hz}$ are believed to be environmental. The broad, low amplitude feature around $100-200 \mathrm{~Hz}$ is dependent on the DC power supply used and is believed to be instrumental due to poor power supply noise rejection.

The effective noise bandwidth (ENBW) value shown in the Figs. 10 and 11 is the scaling factor, as defined by Heinzel et al. (2002), allowing the presented amplitude spectra to be converted into power spectral density via

Amplitude Spectrum $=\sqrt{\text { Power Spectral Density } \times \text { ENBW }}$.

\subsection{Instrument noise floor}

Figure 12 shows a long-period average power spectral density plot taken in the magnetically quiet NRCan environment. The prototype instrument resolves the the sub-10pT/ $\sqrt{\mathrm{Hz}}$ RMS at $1 \mathrm{~Hz}$ noise of the sensor core and is consistent with the previous CASSIOPE ePOP MGF results. Significantly, the new digital design achieves the same noise floor and better resolution while providing $900 \mathrm{sps}$ compared to $160 \mathrm{sps}$ in the previous design. The double-peaked noise feature between 200 and $300 \mathrm{~Hz}$ is again believed to be related to the power converter and will be addressed in the next revision of hardware.

\subsection{Electronics noise floor}

Figure 13 shows a long-period average power spectral density plot taken with the fluxgate sensor out of circuit. Note how the noise resulting from the custom PWM 


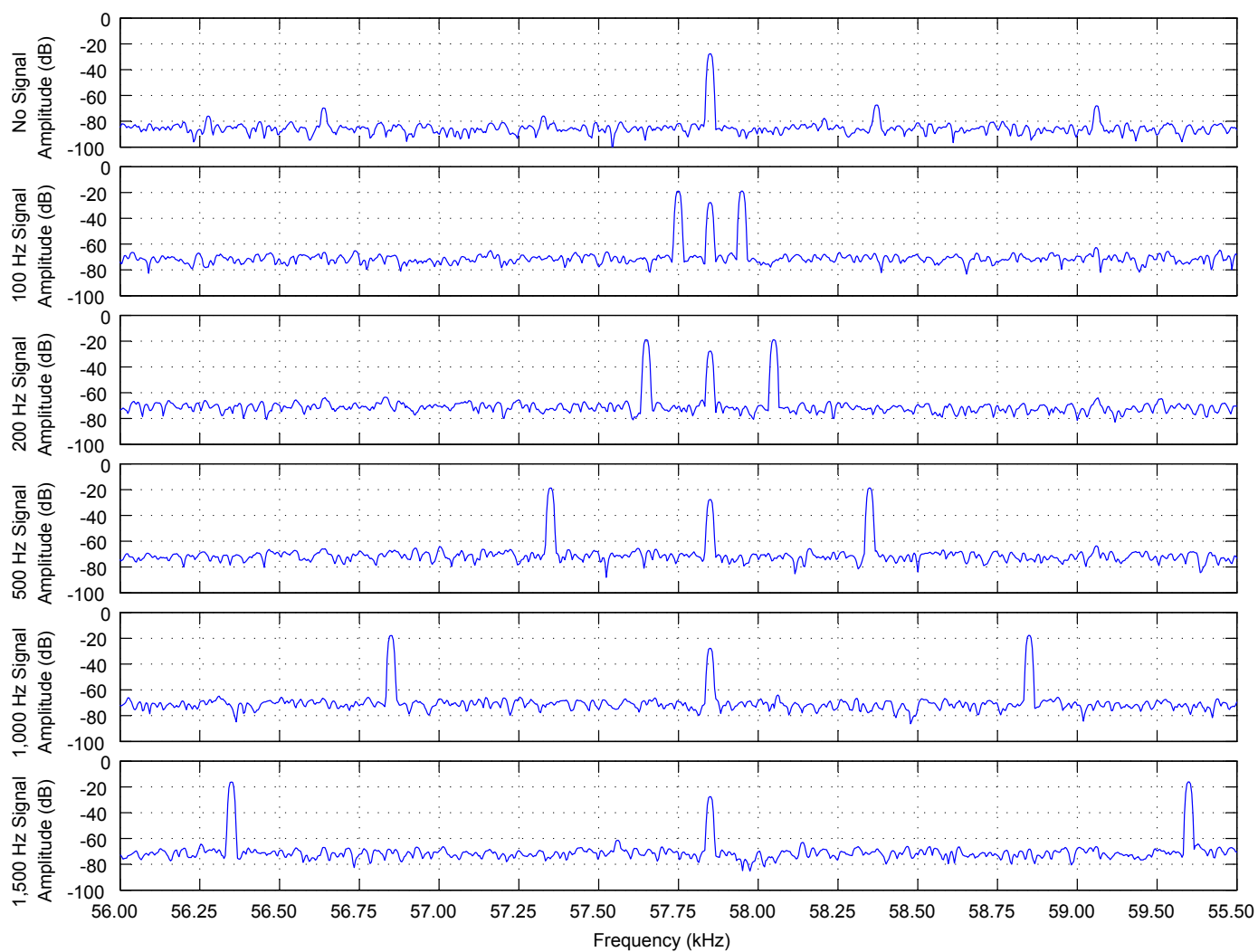

Fig. 8. Spectral analysis of the directly sampled error signal showing the modulation of the $2 f$ major carrier sidebands by applied sinusoidal magnetic signals up to $1500 \mathrm{~Hz}$.

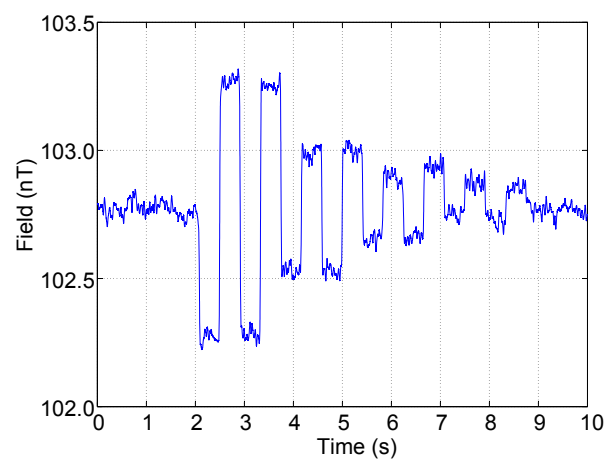

Fig. 9. Time series showing two cycles, each of square waves at amplitudes of $1,0.5,0.25$, and $0.125 \mathrm{nT}$ respectively. Data is post-processed with a $1 \mathrm{~Hz}$ low-pass filter to remove environmental $60 \mathrm{~Hz}$ noise.

digital-to-analog converter and the analog-to-digital converter is well below that measured with the fluxgate sensor in circuit.

\subsection{RMS error}

To quantify the RMS error, the sensor was placed within a known field generated by the Helmholtz coil test equipment

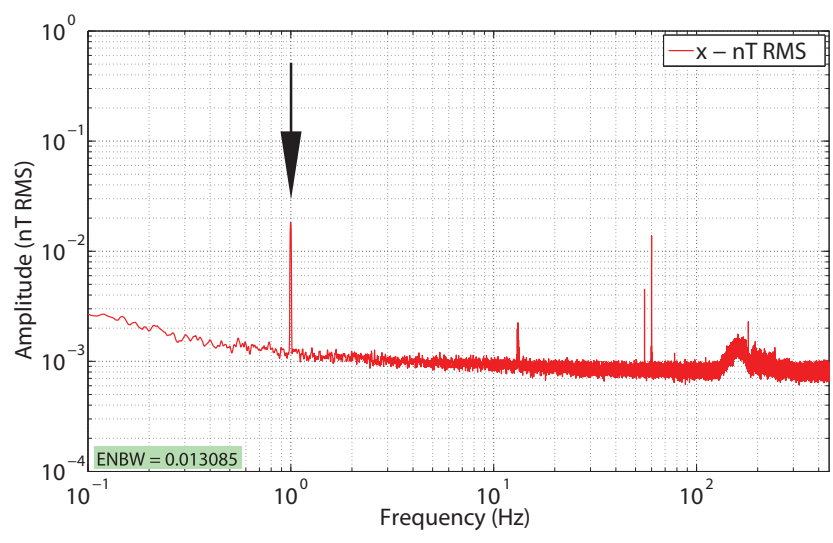

Fig. 10. Amplitude spectrum showing resolution of prototype instrument. Arrow shows an 18 pT RMS sinusoidal test signal at $1 \mathrm{~Hz}$.

at NRCan. Each component was varied over the full-scale range and compared to a measurement of the current used to drive the Helmholtz coil.

The RMS error provides an estimate of the expected absolute error of the instrument at any point in its $\pm 65536 \mathrm{nT}$ range. It is calculated by using the known absolute current measurements to create a set of ideal instrument 


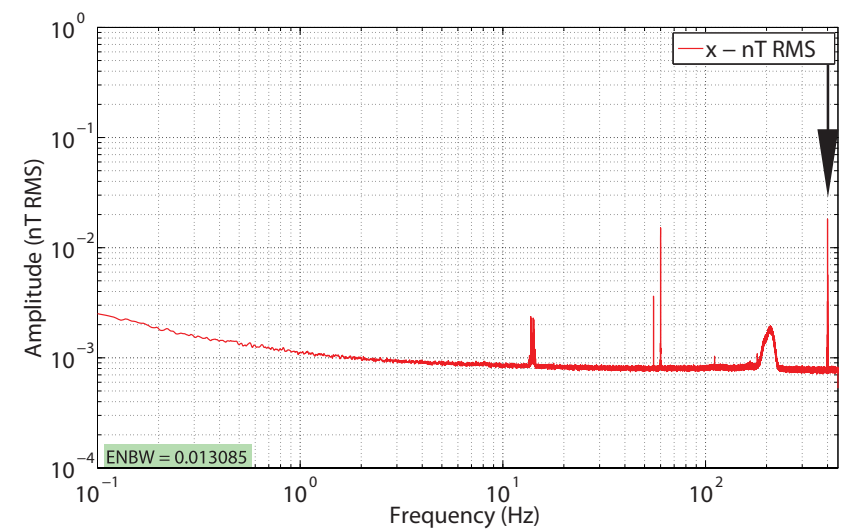

Fig. 11. Amplitude spectrum showing resolution of prototype instrument. Arrow shows an $18 \mathrm{pT}$ RMS sinusoidal test signal at $400 \mathrm{~Hz}$.

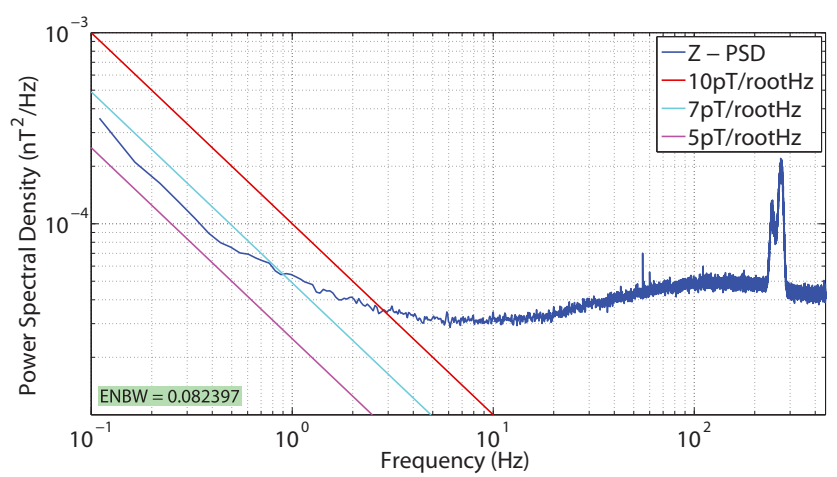

Fig. 12. Power spectral density noise floor of the prototype instrument.

measurements. The difference between each measured point and the ideal measurement are then averaged by a RMS calculation following the equation

RMS Deviation $=\sqrt{\frac{\sum_{i=1}^{n}\left(\operatorname{Ideal}_{n}-\mathrm{Actual}_{n}\right)^{2}}{n}}$.

Each datum is taken as the arithmetic mean of 900 samples (1 s) to cancel out the $60 \mathrm{~Hz}$ contamination. These analyses indicate that the prototype has an RMS error of $6.5 \mathrm{nT}$ ( $X$ component), $6.4 \mathrm{nT}$ ( $Y$ component) and $5.5 \mathrm{nT}$ ( $Z$ component) RMS per point. This is equivalent to

$$
\frac{7 \mathrm{nT}}{ \pm 65536 \mathrm{nT}}=0.00005
$$

or $0.005 \%$ of full scale which meets the mission requirement of $0.1 \%$.

\subsection{Frequency response}

Figure 14 shows the amplitude and phase frequency response of the instrument. Note the peak in amplitude response

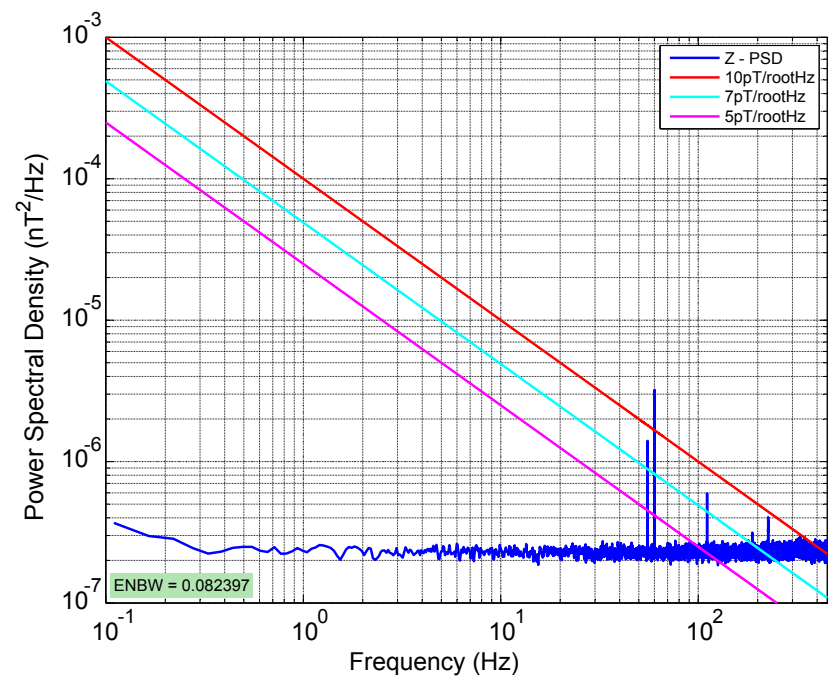

Fig. 13. Power spectral density noise floor of the electronics with the fluxgate sensor out of circuit.

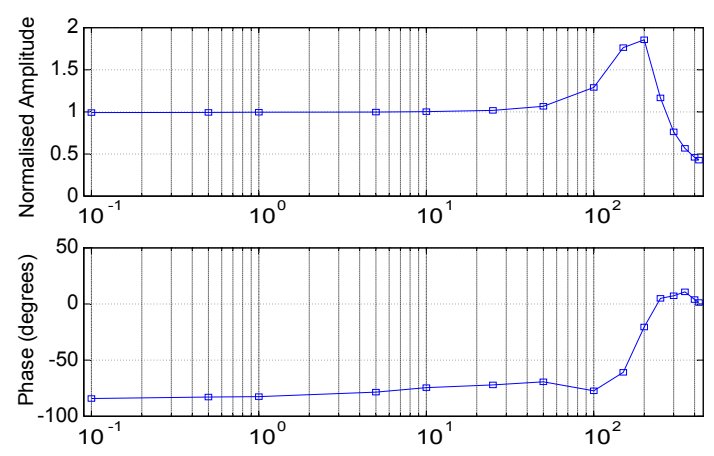

Fig. 14. Amplitude and phase frequency response of the prototype.

around $200 \mathrm{~Hz}$ and the phase shift starting at about $100 \mathrm{~Hz}$. Several factors influence this including the three-pole lowpass filter in the feedback loop, the non-zero pickup of the $\frac{\mathrm{d} \boldsymbol{H}}{\mathrm{d} t}$ action of the sensor, and the vulnerability to aliasing due to the absence of a bandpass filter at $2 f$.

\subsection{Performance summary}

The key performance parameters of the prototype instrument are summarised in Table 2.

\section{Future work}

The current prototype was constructed from multiple printed circuit boards with wiring interconnects and significant debugging hardware. The next hardware iteration will be integrated into a single, flight ready card and the power consumption will be re-tested with the debugging tools removed. The instrument's power supply and the power supply rejection ratio will be improved to remove the double-peaked noise 
Table 2. Performance summary of the prototype instrument.

\begin{tabular}{ll}
\hline Parameter & Current value \\
\hline Resolution & $8 \mathrm{pT}$ \\
Cadence & $900 \mathrm{sps}$ \\
Noise floor & $<10 \mathrm{pT} / \sqrt{\mathrm{Hz}}$ at $1 \mathrm{~Hz}$ \\
RMS error & $0.005 \%$ \\
Potential radiation tolerance & $100 \mathrm{krad}$ \\
Power & $1.5 \mathrm{~W}$ \\
Electronics dimensions & $150 \times 150 \times 30 \mathrm{~mm}$ \\
\hline
\end{tabular}

feature between 100 and $300 \mathrm{~Hz}$ seen in the amplitude and power spectral density plots. Long-term drift tests and offset stability tests versus varying sensor and electronics temperatures will also be completed. A faster analog switch or a third PWM on each component will be added to increase the speed of the digital feedback loop and the bandwidth of the instrument. Faster digital feedback may also require a more sophisticated control algorithm to allow the instrument to track very high slew rates such as those found on spinning sounding rocket applications which can be on the order of $5 \mathrm{~Hz}$.

The prototype instrument is currently part of a proposed secondary plasma science payload on Canada's flagship Polar Communication and Weather (PCW) satellite constellation (Trishchenko and Garand, 2012). The instrument is also expected to be flown as a Canadian contribution to the fourth Norwegian Investigation of Cusp Irregularities (ICI-4) suborbital sounding rocket mission (Moen et al., 2003).

\section{Conclusions}

The prototype instrument delivers $8 \mathrm{pT}$ resolution in a $\pm 65536 \mathrm{nT}$ field at $900 \mathrm{sps}$ with a magnetic noise of less than $10 \mathrm{pT} / \sqrt{\mathrm{Hz}} \mathrm{RMS}$ at $1 \mathrm{~Hz}$. The magnetometer is designed to be radiation tolerant to $100 \mathrm{krad}$ and to be entirely constructed from space grade (Class S) parts so that it can be deployed in extreme radiation environments such as Earth's radiation belts. The instrument uses a novel digital feedback process to improve the sampling rate and resolution while reducing complexity, parts count, and physical size. Significantly, the novel magnetic feedback design combines digital feedback with classic analog temperature compensation. This has produced a prototype modern, digital fluxgate magnetometer suitable for a variety of ground, suborbital, and space applications.

Acknowledgements. This paper describes the improvement, extension and modernisation of an instrument design developed by Magnametrics, Narod Geophysics Ltd, and Bennest Enterprises Ltd for the CASSIOPE/ePOP satellite. The University of Alberta, Department of Physics Electronics Shop provided significant assistance with the digital electronics. The authors would like to acknowledge the assistance of David Barona,
Bill Burris, Miroslaw Ciurzynski, Paul Davis, Alex Degeling, Andy Kale, Shengli Liu, Kyle Murphy, Barry Narod, Louis Ozeke, Jonathan Rae, Don Wallis, and Clare Watt. Instrument development was undertaken with the financial support of the Canadian Space Agency. I. R. Mann is supported by a Discovery Grant from the Canadian NSERC. The authors would like to thank Natural Resources Canada for the use of the Geomagnetics Laboratory facilities and their assistance characterising and calibrating the instrument.

Edited by: H. Svedhem

\section{References}

Acuña, M. H.: Fluxgate magnetometers for outer planets exploration, IEEE T. Mag., 10, 519-523, 1974.

Acuña, M. H. and Ness, N. F.: The Pioneer XI high field fluxgate magnetometer, Technical report, Goddard Space Flight Center, Greenbelt, Maryland, USA, 1973.

Acuña, M. H. and Pellerin, C. J.: A miniature two-axis fluxgate magnetometer, IEEE T. Geosci. Electron., 7, 252-260, 1969.

Acuña, M. H., Scearce, C. S., Seek, J., and Scheifele, J.: The MAGSAT vector magnetometer: A precision fluxgate magnetometer for the measurement of the geomagnetic field, Technical report, National Aeronautics and Space Administration, Goddard Space Flight Center, Greenbelt, Maryland, 1978.

Auster, H., Glassmeier, K., Magnes, W., Aydogar, O., Baumjohann, W., Constantinescu, D., Fischer, D., Fornacon, K., Georgescu, E., Harvey, P., Hillenmaier, O., Kroth, R., Ludlam, M., Narita, Y., and Nakamura, R.: The THEMIS Fluxgate Magnetometer, Space Sci. Rev., 141, 235, 2008.

Carr, C., Brown, P., Zhang, T. L., Gloag, J., Horbury, T., Lucek, E., Magnes, W., O’Brien, H., Oddy, T., Auster, U., Austin, P., Aydogar, O., Balogh, A., Baumjohann, W., Beek, T., Eichelberger, H., Fornacon, K.-H., Georgescu, E., Glassmeier, K.-H., Ludlam, M., Nakamura, R., and Richter, I.: The Double Star magnetic field investigation: instrument design, performance and highlights of the first year's observations, Ann. Geophys., 23, 2713-2732, doi:10.5194/angeo-23-2713-2005, 2005.

Eaton, D. W., Adams, J., Asudeh, I., Atkinson, G. M., Bostock, M. G., Cassidy, J. F., Ferguson, I. J., Samson, C., Snyder, D. B., Tiampo, K. F., and Unsworth, M. J.: Investigating Canada's Lithosphere and earthquake hazards with portable arrays, EOS, 86, 169-173, 2005.

Forslund, A., Belyayev, S., Ivchenko, N., Olsson, G., Edberg, T., Marusenkov, A.: Miniaturized digital fluxgate magnetometer for small spacecraft applications, Measurement Sci. Technol., 19, 015202, doi:10.1088/0957-0233/19/1/015202, 2008.

Friedel, R. H. W., Reeves, G. D., and Obara, T.: Relativistic electron dynamics in the inner magnetosphere - A review, J. Atmos. Sol.Terr. Phys., 64, 265-282, 2002.

Heinzel, G., Rudiger, A., and Schilling, R.: Spectrum and spectral density estimation by the discrete fourier transform (DFT), including a comprehensive list of window functions and some new flat-top windows, Technical report, Max-Planck-Institut fur Gravitationsphysik, Teilinstitut Hannover, 2002.

Luehr, H., Kloecker, N., Oelschlaegel, W., Haeusler, B., and Acuña, M.: The IRM fluxgate magnetometer, IEEE T. Geosci. Remote, 23, 259-261, 1985. 
Magnes, W., Pierce, D., Valavanoglou, A., Means, J., Baumjohann, W., Russell, C. T., Schwingenschuh, K., and Graber, G.: A sigmadelta fluxgate magnetometer for space applications, Measurement Sci. Technol., 14, 1003-1012, doi:10.1088/09570233/14/7/314, 2003.

Magnes, W., Oberst, M., Valavanoglou, A., Hauer, H., Hagen, C., Jernej, I., Neubauer, H., Baumjohann, W., Pierce, D., Means, J., and Falkner, P.: Highly integrated front-end electronics for spaceborne fluxgate sensors, Measurement Sci. Technol., 19, 115801, doi:10.1088/0957-0233/19/11/115801, 2008.

Mann, I. R., Balmain, K. G., Blake, J. B., Boteler, D., Bourdarie, S., Clemmons, J. H., Dent, Z. C., Degeling, A. W., Fedosejeves, R., Fennell, J. F., Fraser, B. J., Green, J. C., Jordanova, V. K., Kale, A., Kistler, L. M., Knudsen, D. J., Lessard, M. R., Loto'aniu, T. M., Milling, D. K., O’Brien, T. P., Onsager, T. G., Ozeke, L. G., Rae, I. J., Rankin, R., Reeves, G. D., Ridley, A. J., Sofko, G. J., Summers, D., Thomson, I., Thorne, R. M., Tsui, Y. Y., Unick, C., Vassiliadis, D., Wygant, J. R., and Yau, A. W.: The outer radiation belt injection, transport, acceleration and loss satellite (ORBITALS): A Canadian small satellite mission for ILWS, Adv. Space Res., 38, 1838-1860, doi:10.1016/j.asr.2005.11.009, 2006.

Mann, I. R., Milling, D. K., Rae, I. J., Ozeke, L. O., Kale, A., Kale, Z., Murphy, K., Parent, A., Usanova, M., Pahud, D., Lee, E.A., Amalraj, V., Wallis, D., Angelopoulos, V., Glassmeier, K.H., Russell, C., Auster, H.-U., and Singer, H.: The upgraded CARISMA magnetometer array in the THEMIS era, Space Sci. Rev., 141, 413-451, doi:10.1007/s11214-008-9457-6, 2008.

Mann, I. R., Rae, I. J., Ozeke, L. G., Miles, D. M., and Yau, A. W.: Plasma and Radiation In Molniya Orbit (PRIMO) Science Objective and User's Needs Definition Document, Technical report, Universities of Alberta and Calgary for the Canadian Space Agency, University of Alberta, Department of Physics, Edmonton, Canada, 2011.

Moen, J., Bekkeng, J. K., Pedersen, A., Aase, J. G., de Feraudy, H., Søraas, F., Blix, T. A., Lester, M., and Pryse, S. E.: ICI-1: a new sounding rocket concept to observe micro-scale physics in the cusp ionosphere, in: 16th ESA Symposium on European Rocket and Balloon Programmes and Related Research, 2-5 June 2003, Sankt Gallen, Switzerland, edited by: Warmbein, B., ESA Publications Division SP 530, Noordwijk, 543-548, 2003.
Narod, B.: The origin of noise and hysteresis in permalloy ring-core fluxgate sensors, EGU General Assembly Conference Abstracts, vol. 15, p. 2357, 2013.

Narod, B. B. and Bennest, J. R.: Ring-core fluxgate magnetometers for use as observatory variometers, Phys. Earth Planet. Interior., 59, 23-28, doi:10.1016/0031-9201(90)90205-C, 1990.

O'Brien, H., Brown, P., Beek, T., Carr, C., Cupido, E., and Oddy, T.: A radiation tolerant digital fluxgate magnetometer, Meas. Sci. Technol., 18, 3645, doi:10.1088/0957-0233/18/11/050, 2007.

Pedersen, E. B., Primdahl, F., Petersen, J. R., Merayo, J. M. G., Brauer, P., and Nielsen, O. V.: Digital fluxgate magnetometer for the Astrid-2 satellite, Measurement Sci. Technol., 10, N124N129, doi:10.1088/0957-0233/10/11/402, 1999.

Primdahl, F.: Temperature compensation of fluxgate magnetometers, IEEE T. Mag., 6.4, 819-822, 1970.

Primdahl, F., Nielsen, O., Petersen, J., and Ripka, P.: High frequency fluxgate sensor noise, Electron. Lett., 30, 481-482, 1994.

Ripka, P.: Magnetic Sensors and Magnetometers, Artech House, Inc., Northwood, MA, 2001.

Schultz, A.: EMScope: A continental scale magnetotelluric observatory and data discovery resource, Data Sci. J., 8, IGY6-IGY20, 2009.

Seppälä, A., Verronen, P. T., Clilverd, M. A., Randall, C. E., Tamminen, J., Sofieva, V., Backman, L., and Kyrölä, E.: Arctic and Antarctic polar winter $\mathrm{NO}_{\mathrm{x}}$ and energetic particle precipitation in 2002-2006, Geophys. Res. Lett., 34, L12810, doi:10.1029/2007GL029733, 2007.

Singer, H. J., Sullivan, W. P., Anderson, P., Mozer, F., Harvey, P., Wygant, J., and McNeil, W.: Fluxgate magnetometer instrument on the CRRES, J. Space Rocket., 29, 599-601, 1992.

Trishchenko, A. P. and Garand, L.: Observing polar regions from space: advantages of a satellite system on a highly elliptical orbit versus a constellation of low Earth polar orbiters, Can. J. Remote Sens., 38, 12-24, 2012.

Wallis, D. D., Narod, B. B., and Bennest, J. R.: The ePOP/CASSIOPE Magnetic Field Instrument, Proceedings of the 13th Canadian Astronautics Conference, ASTRO 2006, organized by CASI - Canadian Astronautics and Space Institute, 25-27 April 2006, Montreal, QC, Canada, 2006. 